\title{
Interface engineering of tungsten carbide/phosphide heterostructures anchored on N,P-codoped carbon for high-efficiency hydrogen evolution reaction
}

\author{
Hongfang $\mathrm{Du}^{1 \dagger}$, Zhuzhu $\mathrm{Du}^{1 \dagger}$, Tingfeng Wang ${ }^{1}$, Song $\mathrm{He}^{1}$, Kai Yang ${ }^{1}$, Ke Wang ${ }^{1}$, Linghai Xie ${ }^{2}$, Wei $\mathrm{Ai}^{{ }^{*}}$ \\ and Wei Huang ${ }^{1,2,3^{*}}$
}

\begin{abstract}
The development of inexpensive and efficient Pt-free electrocatalysts for the hydrogen evolution reaction (HER) is greatly crucial for water electrolysis. Tungsten carbide (WC) exhibiting a Pt-like electronic structure represents an attractive alternative, although its overall performance is limited by the strong $\mathrm{W}-\mathrm{H}$ bond that impedes hydrogen desorption. Here, we employed an in-situ interface engineering strategy to construct high-performance and cost effective electrocatalysts comprising WC/tungsten phosphide (WP) heterostructures that were anchored on $\mathrm{N}, \mathrm{P}$-codoped carbon (WC/WP@NPC) via a one-step pyrolysis of a melamine polyphosphate/ $\mathrm{WO}_{3}$ hybrid in an inert atmosphere. Owing to the crystal structure compatibility and electron-rich property of WP, it optimizes the electronic structure and hydrogen adsorption configuration of $\mathrm{WC}$, thus significantly weakening the $\mathrm{W}-\mathrm{H}$ bond with a thermoneutral Gibbs free energy of hydrogen adsorption $\left(\Delta G_{\mathrm{H}^{*}}\right)$ of $-0.05 \mathrm{eV}$. Additionally, NPC ensures fast electron transport and structural stability of the WC/WP@NPC ternary architecture. These synergistically lead to outstanding HER performances of the catalyst in acidic and alkaline media. Our finding offers a new strategy for designing Pt-alternative electrocatalysts with outstanding electrochemical performances for high-efficiency water splitting and other applications.
\end{abstract}

Keywords: WC, WP, heterostructure, interface engineering, hydrogen evolution reaction

\section{INTRODUCTION}

Hydrogen, a sustainable and environmentally benign energy carrier, is being extensively investigated as a promising fossilfuel alternative $[1,2]$. Water splitting employing earth-abundant water resources and renewable electrical energy is among the simplest and most ecofriendly approaches for producing hydrogen $[3,4]$. However, electrolyzers strongly require noblemetal electrocatalysts, which are scarce and expensive, to drive water splitting because of the sluggish electrode kinetics [5]. For example, Pt-based electrocatalysts are required to achieve a high-efficiency hydrogen evolution reaction (HER), which is the cathodic half-reaction in water splitting [6]. Thus, it is imperative to develop cost-effective HER catalysts with high activity to substitute the traditional Pt-based ones [7-9]. Thus far, transition-metal-based compounds ( $\mathrm{Fe}, \mathrm{Co}, \mathrm{Ni}, \mathrm{Mo}, \mathrm{W}$, etc.) have been identified as promising electrocatalysts for HER. Among them, W-based materials are highly desirable for practical application because of their inexpensiveness and attractive electrocatalytic activities [10].

Further, tungsten carbide (WC) has emerged as a noble metal alternative because of its Pt-like electronic structure and catalytic behaviors in hydrogenolysis [11-14]. Unfortunately, WC does not behave like Pt in HER because of its potent affinity to the hydrogen intermediate, which is disadvantageous to hydrogen desorption [14]. Thus, the tuning of the electronic structure of WC via doping [15,16], hybridization [17-20], or heterostructuring [21-24] is an effective approach for weakening the $\mathrm{W}-\mathrm{H}$ bond to facilitate HER. Specifically, heterostructuring via employing other transition metal compounds generates heterointerfaces that can redistribute electrons, which would synergistically enhance the electrocatalytic activity of WC [25]. Consequently, WC-based heterostructures, including $\mathrm{W}_{2} \mathrm{C} / \mathrm{WC}$ $[21,23,26,27], \mathrm{Mo}_{2} \mathrm{C} / \mathrm{WC}[24], \mathrm{W}_{2} \mathrm{~N} / \mathrm{WC}[6,28], \mathrm{a}-\mathrm{WO}_{x} / \mathrm{WC}$ [22], and $\mathrm{WS}_{2} / \mathrm{W}_{x} \mathrm{C}$ [29], have been reported. However, these materials cannot adequately tune the electronic structure of WC because of the mismatches in their physicochemical properties. Fortunately, tungsten phosphide (WP) with high conductivity holds similar building blocks with WC. Fig. 1a, b, as well as Table S1, reveal that WC and WP consist of W trigonal prism units that are selectively occupied by $\mathrm{C}$ or $\mathrm{P}$ atoms at the interstitial positions [30]; the prism of WP is slightly distorted compared with that of WC. Additionally, the electron-rich nature of WP is expected to effectively tune the electronic structure of WC. Therefore, it would be interesting to investigate the catalytic properties of the $\mathrm{WC} / \mathrm{WP}$ heterostructure to

\footnotetext{
${ }^{1}$ Ningbo Institute of Northwestern Polytechnical University (NPU), Frontiers Science Center for Flexible Electronics (FSCFE), Xi'an Institute of Flexible Electronics (IFE) and Xi'an Institute of Biomedical Materials \& Engineering (IBME), NPU, Xi'an 710072, China

${ }^{2}$ Key Laboratory of Organic Electronics and Information Displays (KLOFE) \& Institute of Advanced Materials (IAM), Nanjing University of Posts \& Telecommunications, Nanjing 210023, China

${ }^{3}$ Key Laboratory of Flexible Electronics (KLOFE) \& Institute of Advanced Materials (IAM), Jiangsu National Synergetic Innovation Center for Advanced Materials (SICAM), Nanjing Tech University (NanjingTech), Nanjing 211816, China

$\dagger$ These authors contributed equally to this work.

* Corresponding authors (emails: iamwai@nwpu.edu.cn (Ai W); iamwhuang@nwpu.edu.cn (Huang W))
} 

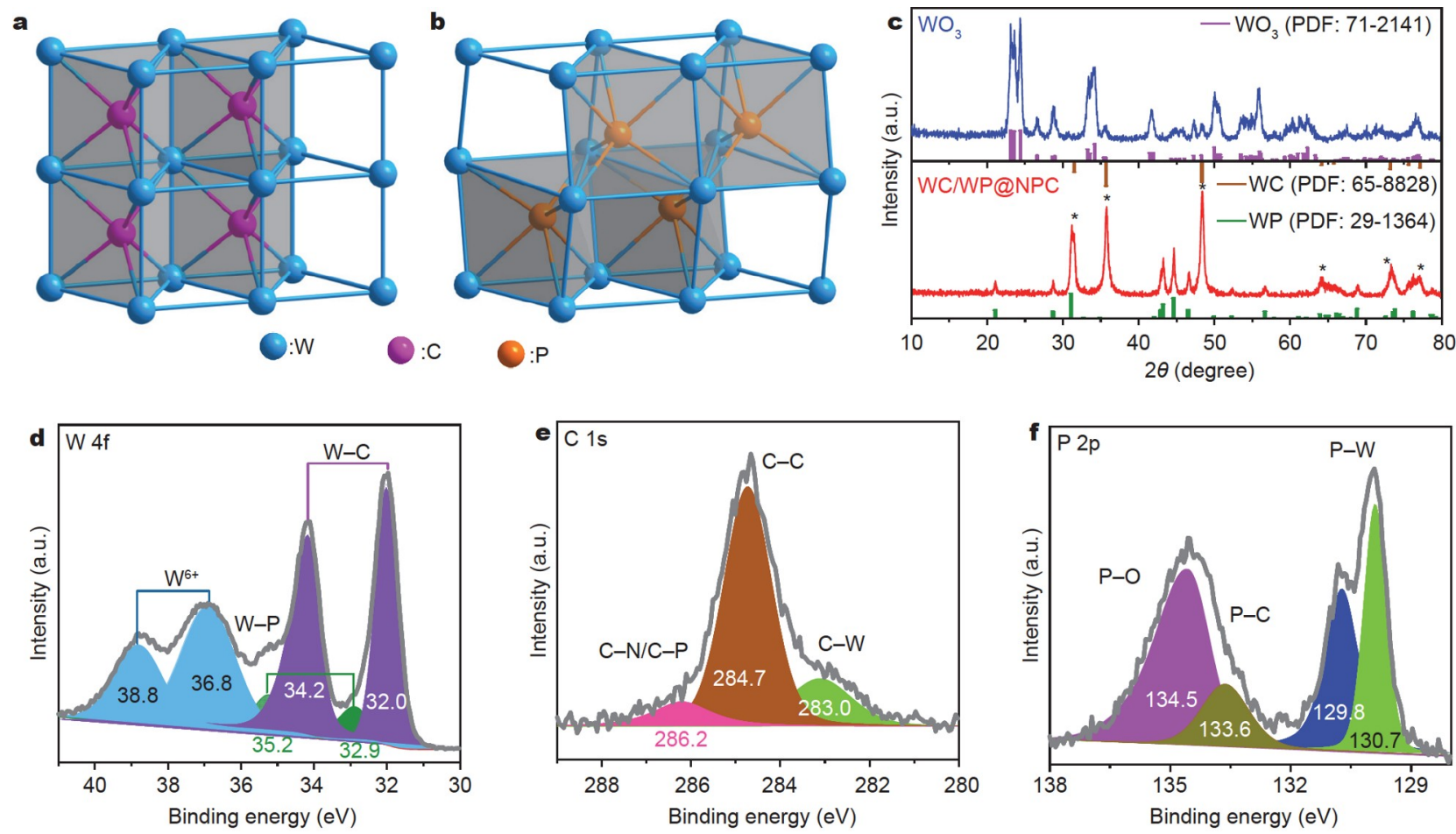

Figure 1 Crystal structures of (a) tungsten carbide (WC; JCPDS No. 619096) and (b) tungsten phosphide (WP; JCPDS No. 42056). (c) XRD patterns of WO and WC/WP@NPC. High-resolution (d) W 4f, (e) C 1s, and (f) P 2p XPS spectra of WC/WP@NPC.

determine how their synergistic effects promote HER performance. To the best of our knowledge, the synthesis of nanostructured WC/WP heterostructure electrocatalysts has not been reported yet.

Herein, we report a simple yet very efficient interface engineering strategy for constructing WC/WP heterostructures anchored on N,P-codoped carbon (WC/WP@NPC) via a onestep pyrolysis process with cost-effective melamine polyphosphate (MPP) and commercial $\mathrm{WO}_{3}$ functioned as the $\mathrm{C} / \mathrm{N} / \mathrm{P}$ and $\mathrm{W}$ sources, respectively. Further, theoretical simulations indicated that WP effectively optimized the hydrogen adsorption configuration of $\mathrm{WC}$, resulting in a favorable Gibbs free energy of hydrogen adsorption $\left(\Delta G_{\mathrm{H}^{*}}\right)$ of $-0.05 \mathrm{eV}$, which was more thermoneutral than that of pure WC $(-0.81 \mathrm{eV})$. In addition, the three-dimensional (3D) NPC matrix, which functioned as a scaffold, ensured fast electron transport and robust structural stability. With these merits, WC/WP@NPC displayed extraordinary HER activity, including a low overpotential of $165 \mathrm{mV}$ to reach $10 \mathrm{~mA} \mathrm{~cm}^{-2}$ and a small Tafel slope of $57 \mathrm{mV} \mathrm{dec}^{-1}$. Moreover, the large-scale production, as well as extensive application of WC/WP@NPC could be achieved because of the simplicity and cost effectiveness of the methodology. This work paves the interface engineering approach for the rational design of high-performance electrocatalysts toward industrial-scale water splitting.

\section{EXPERIMENTAL SECTION}

\section{Synthesis of WC/WP@NPC}

Both MPP and NPC were prepared following the method in our previous study [31]. To synthesize the WC/WP@NPC electrocatalysts, $\mathrm{WO}_{3}$ (Sigma-Aldrich) and MPP powders with mass ratios of $2.5 \%, 5.0 \%$, and $10.0 \%$ were carefully mixed and annealed for $2 \mathrm{~h}$ in an $\mathrm{Ar}$-protected tube furnace at $900^{\circ} \mathrm{C}$ with a ramping rate of $10^{\circ} \mathrm{C} \mathrm{min}^{-1}$ and Ar flow rate of $100 \mathrm{sccm}$ (standard cubic centimeter per minute). After cooling naturally to room temperature, the WC/WP@NPC catalysts were obtained without any posttreatment.

\section{Characterizations}

Scanning electron microscopy (SEM) and energy-dispersive spectroscopy (EDS) analyses were performed on a ZEISS focused ion beam-scanning electron microscope. The Raman spectra were recorded on a WITEC CRM200 Raman system with a $532 \mathrm{~nm}$ laser. Transmission electron microscopy (TEM) measurements were conducted on a JEM-2100 transmission electron microscope at a working voltage of $200 \mathrm{kV}$. The powder X-ray diffraction (XRD) patterns were recorded on a Shimadzu XRD7000 diffractometer with $\mathrm{Cu} \mathrm{Ka}$ radiation $(\lambda=1.5418 \AA$ A $)$. X-ray photoelectron spectroscopy (XPS) was performed on an ESCALAB 250Xi X-ray photoelectron spectrometer employing an $\mathrm{Al} \mathrm{Ka} \mathrm{X}$-ray source $(1486.6 \mathrm{eV}, 15 \mathrm{kV})$.

\section{Electrochemical measurements}

The electrochemical measurements were conducted employing a typical three-electrode system with $0.5 \mathrm{~mol} \mathrm{~L}^{-1} \mathrm{H}_{2} \mathrm{SO}_{4}$ (pH 0.3) or $1 \mathrm{~mol} \mathrm{~L}^{-1} \mathrm{KOH}$ ( $\mathrm{pH} 14$ ) solution as the electrolyte. The saturated calomel electrode (SCE) equipped with a salt bridge, a graphite plate, and glassy carbon electrode (GCE, $\Phi=4.0 \mathrm{~mm}$ ) were served as the reference, counter, and working electrodes, respectively. All the potentials reported herein were converted into the reversible hydrogen electrode (RHE) scale $\left(E_{\mathrm{RHE}}=E_{\mathrm{SCE}}\right.$ $+0.242+0.059 \mathrm{pH}$ ). The catalyst was loaded onto GCE (mass loading was about $0.5 \mathrm{mg} \mathrm{cm}^{-2}$ ) employing Nafion (5wt.\%, Sig- 
ma-Aldrich) as the binder. All the electrochemical data were $i R$ corrected.

\section{Theoretical calculations}

The theoretical calculations were conducted within the density functional theory (DFT) framework employing the Vienna $a b$ initio simulation package $[32,33]$. The generalized gradient approximation was selected for the exchange-correlation potential $[34,35]$. The van der Waals interaction was investigated via Grimme's dispersion-corrected DFT (DFT-D3) [36]. The cutoff energy of the plane wave and energy criterion were set to 400 and $5 \times 10^{-6} \mathrm{eV}$ in the iterative solution of the Kohn-Sham equation. The $\mathrm{p}(3 \times 3) \mathrm{WC}(100)$ and $\mathrm{p}(1 \times 1) \mathrm{WP}(211)$ surfaces were employed as the structural models of WC and WP, respectively. A vacuum thickness of $25 \AA$ was employed to avoid the interactions between the slabs. A $k$-point grid of $3 \times 3 \times 1$ was selected. The WC (100)-WP (211) cluster was constructed as the structural model of the WC/WP heterostructure. All the structures were optimized until the forces on the atoms were $<0.05 \mathrm{eV} / \AA$.

\section{RESULTS AND DISCUSSION}

Fig. S1 schematically shows the synthesis of WC/WP@NPC. Generally, commercial $\mathrm{WO}_{3}$ powder (diameter $=93.4 \mathrm{~nm}$, Fig. S2) and MPP with a mass ratio of $5.0 \%$ were thoroughly ground before they were transferred to the tube furnace. The mixture was annealed to $900^{\circ} \mathrm{C}$ for $2 \mathrm{~h}$ in an Ar atmosphere, after which WC/WP@NPC was obtained without any posttreatment. The decomposition of MPP during annealing released a large amount of $\mathrm{P}$-containing gases, thus triggering the conversion of $\mathrm{WO}_{3}$ into $\mathrm{WPO}_{3-x}(0<x<3)[31,37]$. Subsequently, the interface reaction between $\mathrm{WPO}_{3-x}$ and the MPP-derived porous carbon proceeded via carbothermal reduction, which facilitated the formation of the WC/WP heterostructures anchored on the NPC matrix. Fig. 1c shows that the XRD peaks of the $\mathrm{WO}_{3}$ precursor corresponded well to the monoclinic-type structure (JCPDS No. 71-2141). Interestingly, these peaks disappeared in WC/WP@NPC, and several new diffraction peaks appeared. The peaks at $31.5^{\circ}, 35.7^{\circ}, 48.3^{\circ}, 64.1^{\circ}, 73.2^{\circ}$, and $77.1^{\circ}$ were indexed to WC (JCPDS No. 65-8828) [38], while those at $21.0^{\circ}, 28.7^{\circ}, 31.0^{\circ}, 43.2^{\circ}, 44.6^{\circ}, 46.5^{\circ}, 56.7^{\circ}, 68.8^{\circ}$, and $76.2^{\circ}$ were indexed to WP (JCPDS No. 29-1364) [39], indicating the conversion of $\mathrm{WO}_{3}$ into $\mathrm{WC} / \mathrm{WP}$ heterostructures. The Raman spectra were obtained to further probe the structure of the samples. Fig. S3 shows that the Raman peaks, which corresponded to the stretching $(v)$ and bending $(\delta)$ vibrations of the $\mathrm{O}-\mathrm{W}-\mathrm{O}$ and $\mathrm{W}-\mathrm{O}$ bonds, were detected in the spectrum of $\mathrm{WO}_{3}$ [40]. However, after the annealing treatment with MPP, only two broad peaks were observed at 1348 and $1595 \mathrm{~cm}^{-1}$, and they were sequentially assigned to the $D$ and $G$ bands of the carbonaceous materials [31]. These results once again confirmed the formation of WC/WP@NPC.

The XPS measurements were conducted to analyze the constituents of WC/WP@NPC. The survey XPS spectrum revealed the presence of W, C, P, N, and O elements (Fig. S4). The highresolution $\mathrm{W} 4 \mathrm{f}$ spectrum exhibited six fitting peaks, where the two dominant peaks at 32.0 and $34.2 \mathrm{eV}$ were assigned to $\mathrm{W}-\mathrm{C}$ $[41,42]$, and the pair of small peaks at 32.9 and $35.2 \mathrm{eV}$ were assigned to W-P (Fig. 1d) [43]. Further, the other two peaks at 38.8 and $36.8 \mathrm{eV}$ corresponded to the $\mathrm{W}^{6+}$-based material, which was probably generated via superficial oxidation [44]. The high- resolution $\mathrm{C}$ 1s spectrum (Fig. 1e) could be deconvoluted into three peaks that corresponded to $\mathrm{C}-\mathrm{N}(286.2 \mathrm{eV}), \mathrm{C}-\mathrm{C}$ $(284.7 \mathrm{eV})$, and $\mathrm{C}-\mathrm{W}(283.0 \mathrm{eV})[31,45]$. The $\mathrm{P} 2 \mathrm{p}$ spectrum exhibited four fitting peaks (Fig. 1f): the two peaks at 134.5 and $133.6 \mathrm{eV}$ were assignable to the $\mathrm{P}-\mathrm{O}$ (the phosphate groups) and $\mathrm{P}-\mathrm{C}$ bonds in NPC [46], while the other two at lower binding energies of 130.7 and $129.8 \mathrm{eV}$ corresponded to the $\mathrm{P} 2 \mathrm{p}_{1 / 2}$ and $\mathrm{P} 2 \mathrm{p}_{3 / 2}$ bands of $\mathrm{P}-\mathrm{W}$ [44], respectively. The $\mathrm{N}$ 1s spectrum (Fig. S5a) exhibited two peaks, which were attributable to the graphitic $(401.7 \mathrm{eV})$ and pyridinic $(398.8 \mathrm{eV}) \mathrm{N}$ of NPC [46]. The $\mathrm{O} 1 \mathrm{~s}$ band exhibited four peaks (Fig. S5b), which were assignable to O-P (533.4 eV), O-C (531.9 eV), O-W (531.1 eV), and $\mathrm{O}-\mathrm{H}(530.3 \mathrm{eV})$. Compared with NPC (Fig. S6), the P 2p and $\mathrm{N}$ 1s spectra of WC/WP@NPC shifted to higher binding energies, indicating the transfer of electrons from NPC to the WC/WP heterostructure, which favored HER [47].

Fig. 2a shows the SEM image of WC/WP@NPC exhibiting a moss-like morphology. The high-magnification SEM image (Fig. 2b) reveals that the nanoparticles were uniformly anchored on NPC. Furthermore, the average diameter of the particles was $\sim 68.9 \mathrm{~nm}$ (Fig. S7), which was smaller than that of the $\mathrm{WO}_{3}$ precursor $(93.4 \mathrm{~nm})$. Notably, the morphology of $\mathrm{WC} /$ WP@NPC completely differed from that of NPC (Fig. S8), reflecting the interface reaction between $\mathrm{WPO}_{3-x}$ and NPC. The EDS mapping observations (Fig. 2c) indicate that the W, C, P, N, and $\mathrm{O}$ elements were uniformly distributed in the sample. The TEM image (Fig. 2d) further reveals the dispersion of the nanoparticles on NPC. High-resolution TEM of a randomly selected particle was performed to elucidate the structure of the
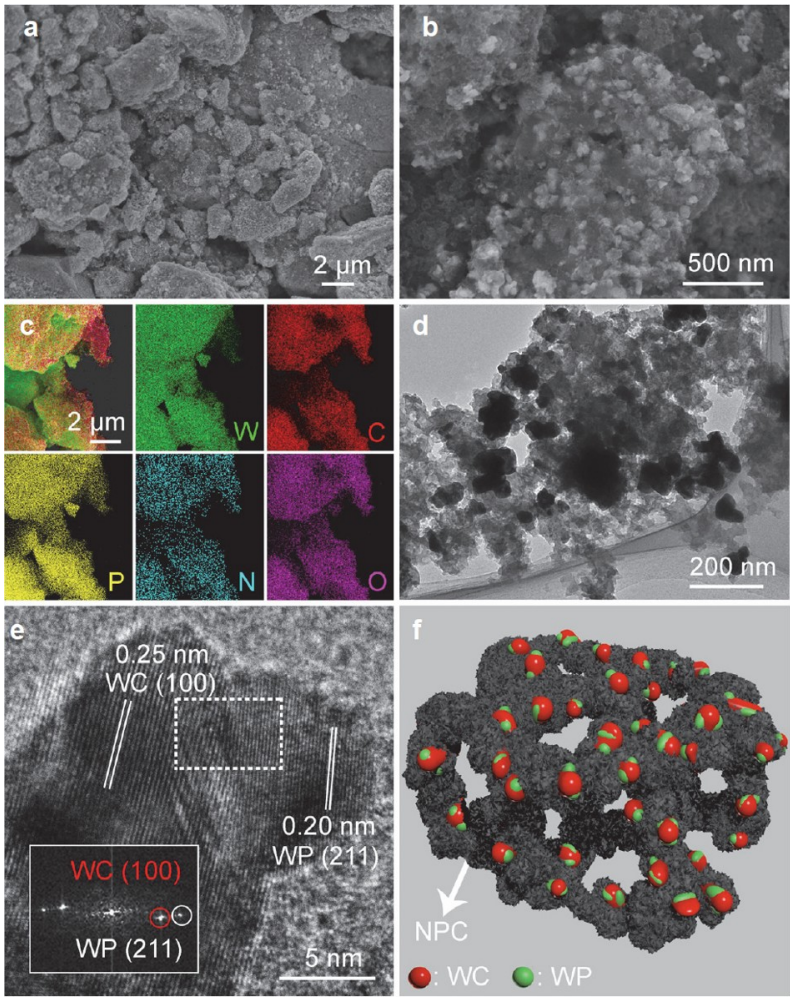

Figure 2 (a, b) SEM images of WC/WP@NPC at different magnifications. (c) EDS mapping, (d) TEM, and (e) high-resolution TEM images of WC/ WP@NPC. The inset of Fig. 2e is the fast Fourier-transform pattern of the WC/WP heterostructures, as acquired from the dash line area. (f) Schematic of WC/WP@NPC. 
catalyst. Fig. 2e shows clear lattice fringes with spacings of 0.25 and $0.20 \mathrm{~nm}$, corresponding to the (100) and (211) planes of WC and WP, respectively [15]. Moreover, the existence of the WC/ WP heterointerfaces was confirmed. Concurrently, the interface of the WC and WP phases was selected for the fast Fourier transform analysis (inset of Fig. 2e), which revealed two pairs of bright diffraction points that stemmed from the WC (100) and WP (211) phases. These results indicate the synthesis of the WC/ WP heterostructures via interface engineering. Fig. $2 \mathrm{f}$ schematically shows the extensive dispersion of the heterostructures on the NPC matrix, forming a 3D interconnected network, which could favor HER.

Considering that the mass ratio of $\mathrm{WO}_{3}$ to MPP could affect the interface reactions, their effects on the properties of the eventual products were evaluated. Fig. S9a shows that when a smaller dosage of $\mathrm{WO}_{3}$ (mass ratio of $\mathrm{WO}_{3}$ to $\mathrm{MPP}=2.5 \%$ ) was utilized, the resulting composite exhibited fewer nanoparticles compared with the original WC/WP@NPC (mass ratio $=5.0 \%$, Fig. 1a, b and Fig. S9b). Upon increasing the mass ratio to $10.0 \%$, large aggregated nanoparticles with a small amount of NPC were detected in the sample (Fig. S9c). The average diameter of the nanoparticles increased to $133.0 \mathrm{~nm}$ (Fig. S10), which was almost two-fold larger than the average diameter of the nanoparticles that were synthesized at a mass ratio of $5.0 \%(68.9 \mathrm{~nm})$. These results indicate that an optimum mass ratio $(5.0 \%)$ could produce a moderate amount of NPC and prevent the aggregation of the WC/WP nanoparticles. The XRD patterns (Fig. S11) confirmed that the materials that were synthesized at mass ratios of $2.5 \%$ and $10.0 \%$ comprised crystalline $\mathrm{WC}$ and $\mathrm{WP}$, as those obtained in the materials synthesized in a mass ratio of $5.0 \%$. The Raman spectra indicated that the peak intensities of the $D$ and $\mathrm{G}$ bands of carbon rapidly decreased with an increase in the mass ratio from $2.5 \%$ to $10.0 \%$ (Fig. S12), and this was attributed to the reduced yield of NPC in the samples, as observed in the SEM images (Fig. S9). This phenomenon is due to the consumption of carbon during the carbothermal reduction process, which further confirms the syntheses of the WC/WP heterostructures via interface engineering.

The electrocatalytic behaviors of WC/WP@NPC toward HER were examined in $0.5 \mathrm{~mol} \mathrm{~L}^{-1} \mathrm{H}_{2} \mathrm{SO}_{4}$ electrolyte with a standard three-electrode system at a scan rate of $2 \mathrm{mV} \mathrm{s}^{-1}$. The corresponding samples were denoted as WC/WP@NPC $(x)$ ( $x$ represents the mass ratio of $\mathrm{WO}_{3}$ to MPP). For further comparison, the benchmark Pt/C (20wt.\%, Johnson Matthey) and NPC were also investigated under the same condition. Expectedly, the $\mathrm{Pt} / \mathrm{C}$ catalyst required an overpotential of $13 \mathrm{mV}$ to reach the current density of $10 \mathrm{~mA} \mathrm{~cm}^{-2}\left(\eta_{10}\right)$, while NPC exhibited negligible electrocatalytic activity (Fig. 3a). Notably, the WC/WP@NPC catalysts exhibited high activity toward HER, where WC/ WP@NPC ${ }_{(5.0)}$ exhibited the most prominent activity with a small $\eta_{10}$ of $165 \mathrm{mV}$, which was lower than those of WC/WP@NPC $(2.5)$ $\left(\eta_{10}=180 \mathrm{mV}\right)$ and WC/WP@NPC $(10.0)\left(\eta_{10}=172 \mathrm{mV}\right)$. The Tafel curves were derived from the corresponding polarization curves and further fitted by the Tafel equation: $\eta=a+b \log (j)$ [48], where $\eta$ is the overpotential, $j$ is the current density, $a$ is a constant, and $b$ is the Tafel slope. Fig. $3 b$ shows that $\mathrm{Pt} / \mathrm{C}$ exhibited a small Tafel slope of $30 \mathrm{mV} \mathrm{dec}^{-1}$, which correlated well with the reported data [15]. The obtained Tafel slopes of WC/WP@NPC $(2.5), W C / W P @ N P C(5.0)$, and WC/WP@NPC $(10.0)$ were 69,57 , and $60 \mathrm{mV} \mathrm{dec}^{-1}$, respectively. This result indicates that HER on WC/WP@NPC proceeded via the Volmer-Heyrovsky mechanism in which the electrochemical desorption
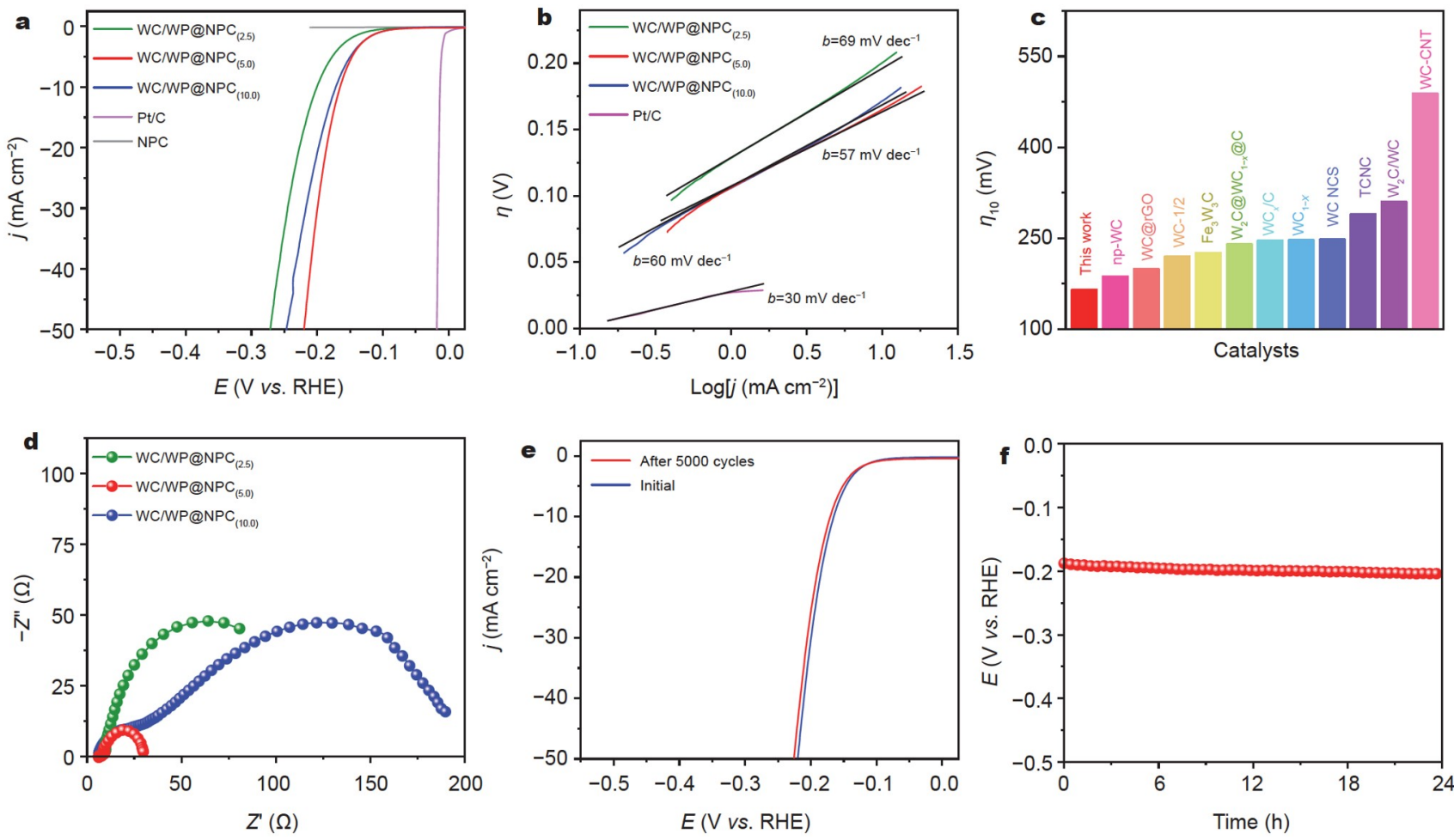

Figure 3 (a) Polarization curves and (b) the corresponding Tafel plots of the samples. (c) $\eta_{10}$ of WC/WP@NPC $(5.0)$ compared with those of recently reported WC-based catalysts. (d) Nyquist plots of the WC/WP@NPC electrodes. (e) Polarization curves of WC/WP@NPC (5.0) before and after 5000 CV cycles. (f) Chronopotentiometric curve of WC/WP@NPC $(5.0)$. 
reaction is the rate-determining step [21]. The lower $\eta_{10}$ and Tafel slope of WC/WP@NPC $(5.0)$ compared with those of the others reflected its superior catalytic activity [49], mainly because of the optimized $\mathrm{WO}_{3} / \mathrm{MPP}$ ratio to produce sufficient $\mathrm{NPC}$ and prevent the aggregation of WC/WP nanoparticles. The electrocatalytic performances of $\mathrm{WC} / \mathrm{WP} @ \mathrm{NPC}_{(5.0)}$ regarding $\eta_{10}$ and the Tafel slope were comparable or even superior to those of most recently reported WC-based HER catalysts (Fig. 3c and Table S2).

To further elucidate the electrode behaviors, electrochemical impedance spectroscopy was performed at an HER overpotential of $200 \mathrm{mV}$ in a frequency range of $0.1-100 \mathrm{kHz}$ employing an alternating current potential amplitude of $5 \mathrm{mV}$. The Nyquist plots (Fig. 3d) revealed that WC/WP@NPC $(10.0)$ exhibited two large arcs, indicating its high resistance toward HER. The arc in the high-frequency region corresponded to the electronic resistance of the catalyst inner-layer/interface, while the arc in the low-frequency region corresponded to the charge-transfer resistance on the electrolyte-catalyst interface [50]. The increases in the electronic conductivity and charge-transfer efficiency of the electrode induce the merging of the two arcs. Thus, WC/ $\mathrm{WP} @ \mathrm{NPC}_{(2.5)}$ and $\mathrm{WC} / \mathrm{WP} @ \mathrm{NPC}_{(5.0)}$ exhibited smaller resistance compared with WC/WP@NPC $(10.0)$, and only one arc was observed. WC/WP@NPC $(5.0)$ exhibited the lowest impedance, which corresponds to superior electrode kinetics for HER. Additionally, NPC also provided paths for bubble release and electrolyte diffusion, thus benefiting mass transport. Notably, despite the negligible HER electrocatalytic activity of NPC, it could still contribute to the excellent electrocatalytic activity of WC/WP@NPC $(5.0)$. For example, the codoped N and P atoms promoted proton adsorption, while the conducting $3 \mathrm{D}$ network facilitated electron transport, both ensuring fast electrode kinetics [51-53]. To evaluate the long-term stability of WC/ WP@NPC $(5.0)$, cyclic voltammetry $(\mathrm{CV})$ tests were performed in a potential range of -0.25 to $0.1 \mathrm{~V}$ at a scan rate of $100 \mathrm{mV} \mathrm{s}^{-1}$. The polarization curves before and after 5000 cycles did not exhibit any significant difference (Fig. 3e), indicating the excellent electrochemical stability of the electrocatalyst. Moreover, no sharp increase was observed in the potential after $24 \mathrm{~h}$ of chronopotentiometric test at a constant current density $\left(20 \mathrm{~mA} \mathrm{~cm}^{-2}\right)$ (Fig. 3f), further verifying the excellent durability of WC/WP@NPC $(5.0)$ toward HER.

In addition to the remarkable HER performance in an acidic medium, the electrocatalytic behaviors of $\mathrm{WC} / \mathrm{WP} @ \mathrm{NPC}_{(5.0)}$ were also evaluated in a basic medium $\left(1.0 \mathrm{~mol} \mathrm{~L}^{-1} \mathrm{KOH}\right.$ solution). Fig. S13 shows that the $\eta_{10}$ and Tafel slope of $\mathrm{WC} /$ WP@NPC $(5.0)$ in the basic medium were almost equal to those in the acidic one, indicating its superior HER activities in both media. Although this WC/WP@NPC $(5.0)$ exhibited a slightly higher Tafel slope $\left(58 \mathrm{mV} \mathrm{dec}^{-1}\right)$ compared with $\mathrm{Pt} / \mathrm{C}$ $\left(38 \mathrm{mV} \mathrm{dec}^{-1}\right)$, the value was among the most active alkaline WC-based electrocatalysts for HER (Table S3). Such impressive HER performances might render WC/WP@NPC $(5.0)$ a promising electrocatalyst for practical application under wide $\mathrm{pH}$ conditions.

To elucidate the high HER activity of WC/WP@NPC, DFT calculations were conducted to investigate $\Delta G_{\mathrm{H}^{*}}$. Considering that the enhancement of the HER activity of WC by heteroatomdoped carbons has been studied $[5,13,38]$, the calculations here only focused on the WC/WP heterostructure. Based on TEM analysis, the WC/WP heterostructure was prepared by com- bining WC (100) and WP (211) surfaces (Fig. S14). For comparison, the WC (100) and WP (211) surfaces were employed as the structural models of WC (Fig. S15) and WP (Fig. S16), respectively. Fig. S17 shows that WC exhibited three probable hydrogen adsorption locations, namely the $\mathrm{W}$ top, $\mathrm{W}-\mathrm{W}$ bridge, and hollow sites. However, after the structural optimization, the hydrogen that was adsorbed onto the top and hollow sites were rearranged into the bridge site (Fig. 4a and Fig. S18a), which is the most stable hydrogen adsorption configuration (denoted as $\left.\mathrm{WC}_{(\mathrm{B}-\mathrm{H})}\right)$. The calculated $\Delta G_{\mathrm{H}^{*}}$ value of $\mathrm{WC}_{(\mathrm{B}-\mathrm{H})}$ was $-0.81 \mathrm{eV}$, which is consistent with the value $(-0.84 \mathrm{eV})$ reported by $\mathrm{Yu}$ et al. [54]. Such a negative $\Delta G_{\mathrm{H}^{*}}$ signified a large barrier for hydrogen desorption during HER owing to the strong $\mathrm{W}-\mathrm{H}$ bond. Regarding WP, the optimized configuration involved the adsorption of hydrogen onto the $\mathrm{P}$ site $\left(\mathrm{WP}_{(\mathrm{P}-\mathrm{H})}\right)$ (Fig. $4 \mathrm{~b}$ and Fig. S18b). The corresponding $\Delta G_{\mathrm{H}^{*}}$ value was calculated as $-0.22 \mathrm{eV}$. Regarding the WC/WP heterostructure, the hydrogen adsorbed on the interfacial bridge site of $\mathrm{WC}\left(\mathrm{WC}_{(\mathrm{B}-\mathrm{H})} / \mathrm{WP}\right)$ and the interfacial $\mathrm{P}$ site of WP $\left(\mathrm{WC} / \mathrm{WP}_{(\mathrm{P}-\mathrm{H})}\right)$ accounted for $\Delta G_{\mathrm{H}^{*}}$ values of -0.63 and $-0.31 \mathrm{eV}$, respectively (Fig. $4 \mathrm{~d}$, e, and Fig. S19). Compared with $\mathrm{WC}_{(\mathrm{B}-\mathrm{H})}$ and $\mathrm{WP}_{(\mathrm{P}-\mathrm{H}) \text {, the changes of }}$ $\Delta G_{\mathrm{H}^{*}}$ in the heterostructure indicated a strong electronic interaction between the two components [29]. Surprisingly, the heterostructure stabilized the hydrogen adsorption on the $\mathrm{W}$ top site of $\mathrm{WC}\left(\mathrm{WC}_{(\mathrm{T}-\mathrm{H})} / \mathrm{WP}\right.$; Fig. $4 \mathrm{c}$ and Fig. S19a). The more thermoneutral $\Delta G_{\mathrm{H}^{*}}$ of $\mathrm{WC}_{(\mathrm{T}-\mathrm{H})} / \mathrm{WP}(-0.05 \mathrm{eV})$ than those of the others indicated the great weakening of the $\mathrm{W}-\mathrm{H}$ bond. Therefore, the superb HER activity of WC/WP@NPC proceeded from the optimized hydrogen adsorption configuration, which promoted the hydrogen desorption kinetics during HER.

\section{CONCLUSIONS}

In summary, WC/WP@NPC electrocatalysts with WC/WP heterostructures anchored on NPC were developed via an

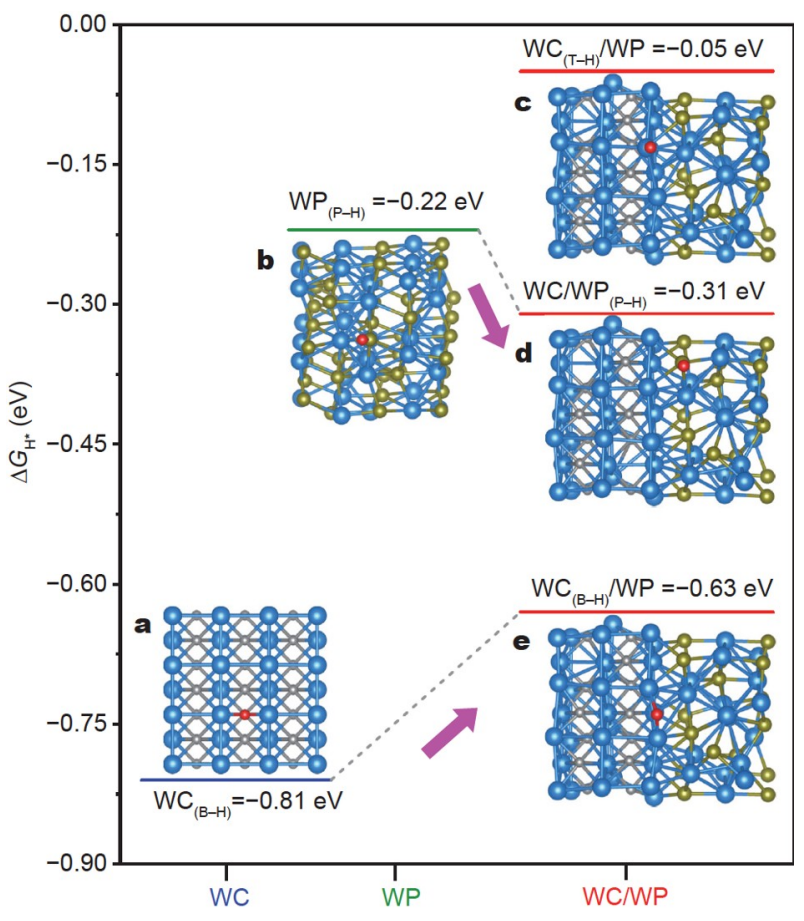

Figure 4 Optimized hydrogen adsorption models and the calculated $\Delta G_{\mathrm{H}^{*}}$ of $\mathrm{WC}, \mathrm{WP}$, and the WC/WP heterostructure. 
interface engineering strategy involving the solid-state transformation reactions between $\mathrm{WO}_{3}$ and MPP. Owing to the structural compatibility and electron-rich feature of WP, it optimized the electronic structure and hydrogen adsorption configuration of WC, thus benefiting the HER process. Via the unique structure and synergistic effects of the ternary components, WC/WP@NPC exhibited enhanced HER kinetics and excellent electrochemical stability toward HER. Moreover, WC/ WP@NPC also demonstrated prominent HER activity over a wide $\mathrm{pH}$ range. The inexpensive, scalable, and facile synthesis combined with the impressive HER performance might endow WC/WP@NPC with great potential for practical application. Notably, this work reveals a new methodology for rationally designing Pt-alternative electrocatalysts with particular heterostructures for HER and other applications.

\section{Received 20 August 2021; accepted 18 October 2021; published online 9 November 2021}

1 Lu XF, Yu L, Zhang J, et al. Ultrafine dual-phased carbide nanocrystals confined in porous nitrogen-doped carbon dodecahedrons for efficient hydrogen evolution reaction. Adv Mater, 2019, 31: 1900699

2 Gao Q, Zhang W, Shi Z, et al. Structural design and electronic modulation of transition-metal-carbide electrocatalysts toward efficient hydrogen evolution. Adv Mater, 2019, 31: 1802880

3 Zhao Y, Yang N, Yao H, et al. Stereodefined codoping of sp-N and S atoms in few-layer graphdiyne for oxygen evolution reaction. J Am Chem Soc, 2019, 141: 7240-7244

4 Shang Z, Zhang Y, Luo L, et al. $\mathrm{MoS}_{x}$ microgrid electrodes with geometric jumping effect for enhancing hydrogen evolution efficiency. Sci China Mater, 2021, 64: 892-898

$5 \mathrm{Li} \mathrm{H}, \mathrm{Hu} \mathrm{M}$, Zhang L, et al. Hybridization of bimetallic molybdenumtungsten carbide with nitrogen-doped carbon: A rational design of super active porous composite nanowires with tailored electronic structure for boosting hydrogen evolution catalysis. Adv Funct Mater, 2020, 30: 2003198

6 Diao J, Qiu Y, Liu S, et al. Interfacial engineering of $\mathrm{W}_{2} \mathrm{~N} / \mathrm{WC}$ heterostructures derived from solid-state synthesis: A highly efficient trifunctional electrocatalyst for ORR, OER, and HER. Adv Mater, 2020, 32: 1905679

7 Xu S, Yang L, Liu YZ, et al. Boosting hydrogen evolution performance by using a plasma-sputtered porous monolithic $\mathrm{W}_{2} \mathrm{C} @ \mathrm{WC}_{1-x} / \mathrm{Mo}$ film electrocatalyst. J Mater Chem A, 2020, 8: 19473-19483

$8 \mathrm{He} \mathrm{S}, \mathrm{Du} \mathrm{H}$, Wang K, et al. Low-temperature molten salt synthesis of $\mathrm{MoS}_{2} @ \mathrm{CoS}_{2}$ heterostructures for efficient hydrogen evolution reaction. Chem Commun, 2020, 56: 5548-5551

9 Wang K, Du H, He S, et al. Kinetically controlled, scalable synthesis of $\gamma$-FeOOH nanosheet arrays on nickel foam toward efficient oxygen evolution: The key role of in-situ-generated $\gamma$-NiOOH. Adv Mater, 2021, 33: 2005587

10 Yu P, Wang F, Shifa TA, et al. Earth abundant materials beyond transition metal dichalcogenides: A focus on electrocatalyzing hydrogen evolution reaction. Nano Energy, 2019, 58: 244-276

11 Pan Y, Zhang C, Lin Y, et al. Electrocatalyst engineering and structureactivity relationship in hydrogen evolution reaction: From nanostructures to single atoms. Sci China Mater, 2020, 63: 921-948

$12 \mathrm{He}$ C, Bo T, Wang B, et al. RGO induced one-dimensional bimetallic carbide nanorods: An efficient and $\mathrm{pH}$-universal hydrogen evolution reaction electrocatalyst. Nano Energy, 2019, 62: 85-93

$13 \mathrm{Xu}$ YT, Xiao X, Ye ZM, et al. Cage-confinement pyrolysis route to ultrasmall tungsten carbide nanoparticles for efficient electrocatalytic hydrogen evolution. J Am Chem Soc, 2017, 139: 5285-5288

14 Hong Q, Li T, Zheng S, et al. Modulating proton binding energy on the tungsten carbide nanowires surfaces for boosting hydrogen evolution in acid. J Energy Chem, 2021, 62: 610-616

15 Han N, Yang KR, Lu Z, et al. Nitrogen-doped tungsten carbide nanoarray as an efficient bifunctional electrocatalyst for water splitting in acid. Nat Commun, 2018, 9: 924

16 Wang L, Li Z, Wang K, et al. Tuning d-band center of tungsten carbide via Mo doping for efficient hydrogen evolution and $\mathrm{Zn}-\mathrm{H}_{2} \mathrm{O}$ cell over a wide pH range. Nano Energy, 2020, 74: 104850

17 Feng Q, Xiong Y, Xie L, et al. Tungsten carbide encapsulated in grapelike N-doped carbon nanospheres: One-step facile synthesis for lowcost and highly active electrocatalysts in proton exchange membrane water electrolyzers. ACS Appl Mater Interfaces, 2019, 11: 25123-25132

18 Ma YY, Lang ZL, Yan LK, et al. Highly efficient hydrogen evolution triggered by a multi-interfacial Ni/WC hybrid electrocatalyst. Energy Environ Sci, 2018, 11: 2114-2123

19 Yao M, Wang B, Sun B, et al. Rational design of self-supported $\mathrm{Cu} @ W C$ core-shell mesoporous nanowires for $\mathrm{pH}$-universal hydrogen evolution reaction. Appl Catal B-Environ, 2021, 280: 119451

20 Jin $\mathrm{H}$, Chen J, Mao S, et al. Transition metal induced the contraction of tungsten carbide lattice as superior hydrogen evolution reaction catalyst. ACS Appl Mater Interfaces, 2018, 10: 22094-22101

21 Chen Z, Gong W, Cong S, et al. Eutectoid-structured $\mathrm{WC} / \mathrm{W}_{2} \mathrm{C}$ heterostructures: A new platform for long-term alkaline hydrogen evolution reaction at low overpotentials. Nano Energy, 2020, 68: 104335

22 Cui Y, Tan X, Xiao K, et al. Tungsten oxide/carbide surface heterojunction catalyst with high hydrogen evolution activity. ACS Energy Lett, 2020, 5: 3560-3568

23 Zhang LN, Ma YY, Lang ZL, et al. Ultrafine cable-like $\mathrm{WC} / \mathrm{W}_{2} \mathrm{C}$ heterojunction nanowires covered by graphitic carbon towards highly efficient electrocatalytic hydrogen evolution. J Mater Chem A, 2018, 6: $15395-15403$

24 Zhang X, Zhu Z, Liang X, et al. Encapsulating dual-phased $\mathrm{Mo}_{2} \mathrm{C}-\mathrm{WC}$ nanocrystals into ultrathin carbon nanosheet assemblies for efficient electrocatalytic hydrogen evolution. Chem Eng J, 2021, 408: 127270

25 He L, Zhang W, Mo Q, et al. Molybdenum carbide-oxide heterostructures: In situ surface reconfiguration toward efficient electrocatalytic hydrogen evolution. Angew Chem Int Ed, 2020, 59: 3544-3548

26 Emin S, Altinkaya C, Semerci A, et al. Tungsten carbide electrocatalysts prepared from metallic tungsten nanoparticles for efficient hydrogen evolution. Appl Catal B-Environ, 2018, 236: 147-153

27 Huo L, Liu B, Gao Z, et al. 0D/2D heterojunctions of molybdenum carbide-tungsten carbide quantum dots/N-doped graphene nanosheets as superior and durable electrocatalysts for hydrogen evolution reaction. J Mater Chem A, 2017, 5: 18494-18501

28 Chen J, Pan A, Zhang W, et al. Melamine-assisted synthesis of ultrafine $\mathrm{Mo}_{2} \mathrm{C} / \mathrm{Mo}_{2} \mathrm{~N} @ \mathrm{~N}$-doped carbon nanofibers for enhanced alkaline hydrogen evolution reaction activity. Sci China Mater, 2021, 64: 11501158

29 Wang $\mathrm{F}, \mathrm{He} \mathrm{P}, \mathrm{Li} \mathrm{Y}$, et al. Interface engineered $\mathrm{W}_{x} \mathrm{C} @ \mathrm{WS}_{2}$ nanostructure for enhanced hydrogen evolution catalysis. Adv Funct Mater, 2017, 27: 1605802

30 Oyama ST, Clark P, Wang X, et al. Structural characterization of tungsten phosphide (WP) hydrotreating catalysts by X-ray absorption spectroscopy and nuclear magnetic resonance spectroscopy. J Phys Chem B, 2002, 106: 1913-1920

31 Ai W, Wang X, Zou C, et al. Molecular-level design of hierarchically porous carbons codoped with nitrogen and phosphorus capable of in situ self-activation for sustainable energy systems. Small, 2017, 13: 1602010

32 Kresse G, Furthmüller J. Efficient iterative schemes for $a b$ initio totalenergy calculations using a plane-wave basis set. Phys Rev B, 1996, 54: $11169-11186$

33 Kresse G, Furthmüller J. Efficiency of ab-initio total energy calculations for metals and semiconductors using a plane-wave basis set. Comput Mater Sci, 1996, 6: 15-50

34 Perdew JP, Burke K, Ernzerhof M. Generalized gradient approximation made simple. Phys Rev Lett, 1996, 77: 3865-3868

35 White JA, Bird DM. Implementation of gradient-corrected exchangecorrelation potentials in Car-Parrinello total-energy calculations. Phys Rev B, 1994, 50: 4954-4957

36 Grimme S, Ehrlich S, Goerigk L. Effect of the damping function in dispersion corrected density functional theory. J Comput Chem, 2011, 32: $1456-1465$ 
37 Ai W, Huang Z, Wu L, et al. High-rate, long cycle-life Li-ion battery anodes enabled by ultrasmall tin-based nanoparticles encapsulation. Energy Storage Mater, 2018, 14: 169-178

38 Ma R, Song E, Zhou Y, et al. Ultrafine WC nanoparticles anchored on co-encased, N-doped carbon nanotubes for efficient hydrogen evolution. Energy Storage Mater, 2017, 6: 104-111

$39 \mathrm{Pu} \mathrm{Z}$, Ya X, Amiinu IS, et al. Ultrasmall tungsten phosphide nanoparticles embedded in nitrogen-doped carbon as a highly active and stable hydrogen-evolution electrocatalyst. J Mater Chem A, 2016, 4: 15327-15332

40 Garcia-Sanchez RF, Ahmido T, Casimir D, et al. Thermal effects associated with the Raman spectroscopy of $\mathrm{WO}_{3}$ gas-sensor materials. J Phys Chem A, 2013, 117: 13825-13831

41 Shi M, Li W, Fang J, et al. Electronic structure tuning during facile construction of two-phase tungsten based electrocatalyst for hydrogen evolution reaction. Electrochim Acta, 2018, 283: 834-841

42 Zhao Z, Zhu Z, Wang F, et al. Bimetallic carbides embedded in heteroatom-doped carbon nanotubes for efficient electrocatalytic hydrogen evolution reaction and high-performance lithium storage. Chem Eng J, 2021, 415: 128885

43 Shen Y, Li L, Xi J, et al. A facile approach to fabricate free-standing hydrogen evolution electrodes: Riveting tungsten carbide nanocrystals to graphite felt fabrics by carbon nanosheets. J Mater Chem A, 2016, 4: $5817-5822$

44 Xing Z, Liu Q, Asiri AM, et al. High-efficiency electrochemical hydrogen evolution catalyzed by tungsten phosphide submicroparticles. ACS Catal, 2015, 5: 145-149

45 Hunt ST, Nimmanwudipong T, Román-Leshkov Y. Engineering nonsintered, metal-terminated tungsten carbide nanoparticles for catalysis. Angew Chem Int Ed, 2014, 53: 5131-5136

46 Ai W, Zhou W, Du Z, et al. Nitrogen and phosphorus codoped hierarchically porous carbon as an efficient sulfur host for Li-S batteries. Energy Storage Mater, 2017, 6: 112-118

47 Jiang $\mathrm{X}$, Jang $\mathrm{H}$, Liu $\mathrm{S}$, et al. The heterostructure of $\mathrm{Ru}_{2} \mathrm{P} / \mathrm{WO}_{3} / \mathrm{NPC}$ synergistically promotes $\mathrm{H}_{2} \mathrm{O}$ dissociation for improved hydrogen evolution. Angew Chem Int Ed, 2021, 60: 4110-4116

48 Zeng M, Chen Y, Li J, et al. 2D WC single crystal embedded in graphene for enhancing hydrogen evolution reaction. Nano Energy, 2017, 33: 356-362

49 Zhao Y, Yang N, Yang N, et al. Boosting hydrogen evolution reaction on few-layer graphdiyne by sp-N and B co-doping. APL Mater, 2021, 9: 071102

50 Xie C, Chen W, Du S, et al. In-situ phase transition of $\mathrm{WO}_{3}$ boosting electron and hydrogen transfer for enhancing hydrogen evolution on Pt. Nano Energy, 2020, 71: 104653

51 Zhang J, Qu L, Shi G, et al. N,P-codoped carbon networks as efficient metal-free bifunctional catalysts for oxygen reduction and hydrogen evolution reactions. Angew Chem Int Ed, 2016, 55: 2230-2234

52 Zheng Y, Jiao Y, Li LH, et al. Toward design of synergistically active carbon-based catalysts for electrocatalytic hydrogen evolution. ACS Nano, 2014, 8: 5290-5296

53 Zhao Y, Yang N, Yu R, et al. Unique structural advances of graphdiyne for energy applications. EnergyChem, 2020, 2: 100041

54 Yu GQ, Huang BY, Chen SM, et al. The combined role of faceting and heteroatom doping for hydrogen evolution on a WC electrocatalyst in aqueous solution: A density functional theory study. J Phys Chem C, 2021, 125: 4602-4613

Acknowledgements This work was financially supported by the National Natural Science Foundation of China (51902261 and 61935017), the Joint Research Funds of the Department of Science \& Technology of Shaanxi Province and NPU (2020GXLH-Z-024), the Natural Science Basic Research Program of Shaanxi Province (2021JQ-096 and 2021JQ-107), the Natural Science Foundation of Ningbo (202003N4053 and 202003N4046), the Fundamental Research Funds for the Central Universities (31020180QD094, 31020180 QD116 and G2021KY05106), and the Start-up Funding from NPU. The authors also thank the Analytical \& Testing Center of NPU for XPS characterizations.
Author contributions $\quad \mathrm{Du} \mathrm{H}$ and $\mathrm{Du} \mathrm{Z}$ performed the experiments and wrote the manuscript; Wang $\mathrm{T}, \mathrm{He} \mathrm{S}$, and Yang $\mathrm{K}$ assisted in the characterizations and electrocatalytic tests; Wang $\mathrm{K}$ and Xie L assisted in the theoretical analysis; $\mathrm{Ai} \mathrm{W}$ designed the project and revised the manuscript; Huang W supervised the project. All authors contributed to the general discussion.

Conflict of interest The authors declare no conflict of interest.

Supplementary information Supporting data are available in the online version of the paper.

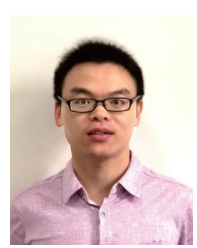

Wei Ai received his $\mathrm{MSc}$ and $\mathrm{PhD}$ degrees from Nanjing University of Posts and Telecommunications and $\mathrm{Na}$ nyang Technological University, respectively. He joined the Institute of Flexible Electronics, Northwestern Polytechnical University in 2018 as a full professor. His research interests are electrochemical materials and technologies, new energy devices, flexible intelligence technologies, etc.

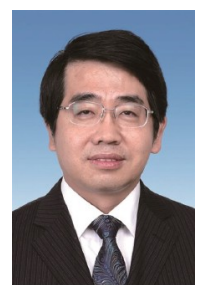

Wei Huang received his BSc, MSc, and $\mathrm{PhD}$ degrees in chemistry from Peking University in 1983, 1988 and 1992, respectively. $\mathrm{He}$ is an academician of the Chinese Academy of Sciences, Foreign Academician and Honorary Doctor of the Russian Academy of Sciences, and a Fellow of the Royal Society of Chemistry. His research interests include organic optoelectronics and flexible electronics.

\section{氮磷共掺杂碳负载WC/WP异质结的电催化析氢性能 研究}

杜洪方 ${ }^{1 \dagger}$, 杜祝祝 ${ }^{1 \dagger}$, 王庭峰 ${ }^{1}$, 何松 ${ }^{1}$, 杨凯 $^{1}$, 王珂 ${ }^{1}$, 解令海 $^{2}$, 艾伟 ${ }^{1^{*}}$, 黄维 $1,2,3^{*}$

摘要 廉价、高效、稳定的非铂析氢催化剂是电解水制氢技术发展的 关键. WC 具有类似铂的电子结构, 且价格低廉, 是极具潜力的析氢催化 剂. 然而, 反应中间体 $\mathrm{W}-\mathrm{H}$ 的结合力强, 不利于 $\mathrm{H}$ 解离, 导致析氢性能不 佳. 针对这一难题, 本工作利用与 $\mathrm{WC}$ 晶体结构兼容性好, 且具有富电子 特性的WP来调控其电子结构, 通过一步原位固相界面反应成功制备了 氮磷共掺杂碳负载WC/WP异质结(WC/WP@NPC)催化剂. 密度泛函理 论计算表明, 该异质结设计有效减小了 $\mathrm{W}-\mathrm{H}$ 键键强, 使材料具有更适中 的氢原子吸附自由能 $(-0.05 \mathrm{eV})$. 得益于 $\mathrm{WC} / \mathrm{WP}$ 异质结与氮磷共掺杂 碳之间的协同增效作用, WC/WP@NPC在酸性和碱性电解质中均表现 出了优异的催化性能. 本工作为高效非铂析氢催化剂的设计制备提供 了新思路. 